\title{
datastructures: An R package for organisation and storage of data
}

\section{DOI: $10.21105 /$ joss. 00910}

\section{Software}

- Review ¿t

- Repository ca

- Archive [u

Submitted: 20 August 2018 Published: 27 August 2018

\section{License}

Authors of papers retain copyright and release the work under a Creative Commons Attribution 4.0 International License (CC-BY).

\section{Simon Dirmeier ${ }^{1}$}

1 Department of Biosystems Science and Engineering, ETH Zurich

\section{Summary}

In computer science, data structures are objects for organisation and storage of data. They are often a key for efficient computation. So far the statistical computing language R (R Core Team, 2018) only supports simple data structures such as lists, matrices and data tables, whereas other data structures such as priority queues, stacks or hashmaps are not supported. These data structures are essential in many computer science and statistics problems, like graph algorithms, string analysis or scheduling. Single packages, for example hashmap (Russell, 2017) or dequer (Schmidt, 2017), have already been proposed, although none of them support a wider range of data structures with a unified interface.

In order to access a richer repertoire of data types, and thus enabling efficient computation of various problems, we propose the $\mathrm{R}$ package datastructures. The package uses Boost and STL data types in a $\mathrm{C}++$ backend and exports these to $\mathrm{R}$ using the Rcpp (Eddelbuettel \& François, 2011) module system.

So far datastructures supports three different groups of data types. Heaps Fibonacci and binomial heaps can be used for priority queue operations, such as in (a version of) Dijkstra's algorithm for finding shortest paths. Maps Hash-, bi- and multimaps are associative arrays that establish key-value relationships. Deques Stacks and queues are linked lists supporting the LIFO or FIFO principle, respectively.

We used an object-oriented approach where every data structure is exported using S4 classes. For easier maintainance and extensibility we heavily rely ond polymorphism and inheritance of the implemented class system.

The package datastructures aims to bridge the gap between statistical and classical programming languages, where usage of these data structures are fairly common. In addition, in the near future we will add support of formats such as suffix arrays and suffix trees, red-black- and B-trees.

\section{Examples}

The following example shows a use case of a hashmap for an artificial data set.

$>$ library(datastructures)

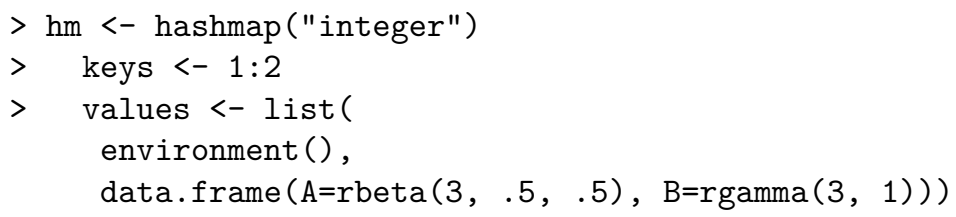


JESS

$>\quad h m[$ keys $]<-$ values

$>\mathrm{hm}[2 \mathrm{~L}]$

[[1] ]

A $\quad$ B

$\begin{array}{lll}1 & 0.5649580 & 1.8091666\end{array}$

$20.2313472 \quad 0.4522518$

30.85333363 .8463516

\section{References}

Eddelbuettel, D., \& François, R. (2011). Rcpp: Seamless R and C++ integration. Journal of Statistical Software, 40(8), 1-18. doi:10.18637/jss.v040.i08

R Core Team. (2018). R: A language and environment for statistical computing. Vienna, Austria: R Foundation for Statistical Computing. Retrieved from https://www.R-project. org/

Russell, N. (2017). Hashmap: The faster hash map. Retrieved from https://CRAN. R-project.org/package=hashmap

Schmidt, D. (2017). dequer: Stacks, queues, and deques for $r$. Retrieved from https: $/ /$ cran.r-project.org/package $=$ dequer 